\title{
Transition from an electron solid to the sequence of fractional quantum Hall states at very low Landau level filling factor
}

\author{
W. Pan ${ }^{1,2}$, H.L. Stormer ${ }^{3,4}$, D.C. Tsui ${ }^{1}$, L.N. Pfeiffer ${ }^{4}$, K.W. Baldwin ${ }^{4}$, and K.W. West ${ }^{4}$ \\ ${ }^{1}$ Department of Electrical Engineering, Princeton University, Princeton, New Jersey 08544 \\ ${ }^{2}$ National High Magnetic Field Laboratory, Tallahassee, Florida 32310 \\ ${ }^{3}$ Department of Physics and Department of Applied Physics, Columbia University, New York, New York 10027 \\ ${ }^{4}$ Bell Labs, Lucent Technologies, Murray Hill, New Jersey 07974
}

(November 2, 2018)

\begin{abstract}
At low Landau level filling of a two-dimensional electron system, typically associated with the formation of an electron crystal, we observe local minima in $R_{x x}$ at filling factors $\nu=2 / 11,3 / 17$, $3 / 19,2 / 13,1 / 7,2 / 15,2 / 17$, and $1 / 9$. Each of these developing fractional quantum Hall (FQHE) states appears only above a filling factor-specific temperature. This can be interpreted as the melting of an electron crystal and subsequent FQHE liquid formation. The observed sequence of FQHE states follow the series of composite fermion states emanating from $\nu=1 / 6$ and $\nu=1 / 8$.
\end{abstract}

The electronic state of a two-dimensional electron system $(2 \mathrm{DES})$ in the presence of a high magnetic $(B)$ field is the result of a competition between electrostatics and subtle quantum mechanics, leading to intricate electronelectron correlations. At extremely high magnetic field and low carrier concentration electrons are forced on tiny orbits as compared to their separation. As a consequence mutual wavefunction overlap vanishes, electrostatics dominates, and an electron crystal, analogous to a classical Wigner crystal of point charges, is expected to form [1-3]. At lower fields, or equivalently higher electron concentration, wavefunction overlap is appreciable. The resulting complex electron-electron correlation leads to the multitude of fractional quantum Hall (FQHE) states [4-7] at rational values of Landau level filling factors $\nu=n / s$, where $n$ is the electron density and $s$ is the magnetic flux density, $s=B / \Phi_{0}$ with the flux quantum $\Phi_{0}=h / e$. The composite fermion (CF) picture [8-11] has been very successful in classifying these FQHE states into series of fractions emanating from neighboring evendenominator $\mathrm{CF}$ liquids. The positive identification of the Wigner crystal remains somewhat controversial, although many different experiments detect behavior that is most naturally explained in terms of such a phase [3]. In particular, in electronic transport experiments the transition to very high resistances is often equated with the onset of electron crystal formation. Such a solid phase is easily pinned by residual potential fluctuations in contrast to the quantum liquids of the FQHE, which can circumvent such obstacles. The weak pinning in the solid phase has been observed in recent microwave measurements [12].

The transition point from the series of FQHE states to the Wigner crystal is one of the oldest questions in the field. Laughlin's original publication on the FQHE estimated this transition to occur around $\nu \sim 1 / 10$ [5] and subsequent calculations refined it to $\nu \sim 1 / 6.5$ [13-17].
Experiment shows a rather more complex behavior. Indeed, even the highest quality specimens - least subject to distortions and hence spurious results from glassy behavior - show at low temperatures a rapid divergence of the resistance for magnetic field values higher than $\nu=1 / 5$. However, even above $\nu=1 / 5$ the resistance diverges in a narrow window $2 / 9 \leq \nu \leq 1 / 5$ [18,19]. This reentrant behavior is interpreted as resulting from complicated, multiple intersections of the non-monotonic ground state energy curve of the FQHE liquid with the smooth equivalent curve for the electron crystal as a function of $\nu$. Also, below $\nu=1 / 5-$ i.e. for higher magnetic fields - the situation is more complex than may be deduced from a single transition point. Weak local minima in the resistance $R_{x x}$ have been observed at filling factors $\nu=1 / 7[20]$ and $\nu=2 / 11$ [21] hinting at the existence of FQHE states in the "forbidden" Wigner crystal regime, although such features always disappear as $T \rightarrow 0$. Furthermore, optical experiments $[22,23]$, performed at elevated temperatures of $\sim 500 \mathrm{mK}$, observe features in the luminescence lines at filling factors $\nu=1 / 7$ and $\nu=1 / 9$. Initially, this has been taken as additional reentrances of the FQHE liquid into the Wigner crystal phase at $\nu=1 / 7$ and $1 / 9$. Later theoretical work [24] interprets the data as those FQHE states representing a higher temperature liquid phase, while the Wigner crystal remains the $T=0$ ground state at $\nu=1 / 7$ and $\nu=1 / 9$. This points again to a much more complex interrelation between Wigner crystal and the FQHE states.

In this paper we present electronic transport data on the ultra-high mobility two-dimensional electron system in a GaAs/AlGaAs quantum well and observe local minima in $R_{x x}$ at a multitude of low Landau level filling factors $\nu=2 / 11,3 / 17,3 / 19,2 / 13,1 / 7,2 / 15,2 / 17$, and $1 / 9$. Each of these developing FQHE states appears only above a filling factor-specific temperature. The FQHE sequence follows the series of composite fermion $(\mathrm{CF})$ 
Landau levels expected to emanate from a $\mathrm{CF}$ liquid at $\nu=1 / 6$ and $\nu=1 / 8$. These observations demonstrate the continued applicability of the $\mathrm{CF}$ model for the FQHE states well into the Wigner crystal regime. The series of FQHE states only exist at elevated temperatures, probably above the point at which the solid has melted.

The quantum well is $500 \AA$ wide and $\delta$-doped symmetrically from both sides at a distance of $2200 \AA$. The electron density of $n \sim 1.0 \times 10^{11} \mathrm{~cm}^{-2}$ and mobility of $\mu \sim 10 \times 10^{6} \mathrm{~cm}^{2} /$ Vsec are established after illumination by a red light emitting diode. At this density, only one electric subband is occupied. The specimen has a size of $5 \mathrm{~mm} \times 5 \mathrm{~mm}$, with 8 indium contacts around its perimeter. The data were taken in dilution refrigerators with different base temperatures placed in three different magnets: $\sim 35 \mathrm{mK}$ in 18 Tesla, $\sim 70 \mathrm{mK}$ in 33 Tesla, and $\sim 70 \mathrm{mK}$ in 42 Tesla. Low frequency $(\sim 3-4 \mathrm{~Hz})$ lockin amplifier techniques were employed to measure $R_{x x}$, with an excitation current of $0.1-1 \mathrm{nA}$ to avoid electron heating.

The high quality of this sample (Fig. 1) is evident from the FQHE sequences $p /(2 p \pm 1)$ showing $R_{x x}$ features up to $\nu=10 / 19$ and $10 / 21$, the sequences $p /(4 p \pm 1)$ showing features up to $\nu=6 / 23$ and $6 / 25$, and the observation of novel FQHE states, e.g. at $\nu=4 / 11$, between $\nu=1 / 3$ and $\nu=2 / 5$. Most remarkable is the flat background around $\nu=1 / 4$. All previous quantum Hall samples, show a rising background starting around $\nu=2 / 7$, indicating that the sample is approaching the insulating regime. No such background is apparent here and $R_{x x}$ at $\nu=1 / 4$ is practically temperature independent as expected for CF liquid at an even-denominator filling factor. This extremely high quality $2 \mathrm{DES}$ allows us to pursue our transport measurement to very low filling factors.

Fig. 2 shows $R_{x x}$ at different temperatures for filling factors $1 / 5 \geq \nu \geq 1 / 7$. In all traces the $\nu=1 / 5$ state is well developed, showing vanishing resistance in $R_{x x}$ and a clean quantized Hall plateau in $R_{x y}$ (not shown). Similar to all high-quality samples, as $T \rightarrow 0, R_{x x}$ diverges for $\nu<1 / 5$ and for $2 / 9<\nu<1 / 5$ (just to the left off the graph). The $80 \mathrm{mK}$ trace clearly shows this divergent behavior. In fact, $R_{x x}$ at $\nu \sim 0.21$ shows activated behavior with a characteristic energy of $1.1 \mathrm{~K}$. This value is very close to those obtained earlier in samples of similar density but with poorer FQHE features and a rising background around $\nu=1 / 4[18,19]$. This seems to suggest that a limiting state has been reached. The re-entrant insulating phase and, by extension, the insulating phase beyond $\nu=1 / 5$ appear to be of intrinsic origin and no longer dominated by appreciable disorder. These data are taken as indicating the formation of a pinned Wigner solid ground state for filling factors $\nu<2 / 9$, interrupted solely by a FQHE state at $\nu=1 / 5$. In particular, for $\nu<1 / 5$ the 2DES becomes insulating and no further
FQHE features are observed for $T \rightarrow 0$. However, raising the temperature to $115 \mathrm{mK}$ through $165 \mathrm{mK}$ uncovers multiple minima in $R_{x x}$ that occur at distinct, rational fractional filling factors $\nu=2 / 11,3 / 17,3 / 19,2 / 13$, and $1 / 7$. They represent the $p /(6 p \pm 1)$ series of FQHE states, emanating from the $\mathrm{CF}$ liquid at $\nu=1 / 6$. The observation of this sequence is a demonstration of the applicability of the CF model even to these very low filling factor FQHE states within the regime of the Wigner solid, albeit at elevated temperatures. Jumping ahead to the data of Figure 3, taken at the yet more extreme conditions of a hybrid magnet, one can even discern features at $\nu=2 / 15,2 / 17$ and $1 / 9$. These are the first representatives of the $p /(8 p \pm 1)$ sequence associated with the $\mathrm{CF}$ liquid state at $\nu=1 / 8$. Both observations attest to the wide applicability of the CF classification scheme.

Returning to Fig. 2 one notices that the resistance values are high compared to typical $R_{x x}$ values in the FQHE regime. They are in the $\mathrm{M} \Omega$ range, rather than the usual $\mathrm{k} \Omega$ range (see Fig. 1). For any temperature, as the magnetic field is raised, $R_{x x}$ becomes increasingly noisy and ultimately collapses [25], taking on even negative values as illustrated in the $135 \mathrm{mK}$ trace. This is accompanied by a sharply increasing out-of-phase component, indicating resistances in the range of $R \sim 1 /(\omega \mathrm{C}) \sim 1 \mathrm{G} \Omega$ (using $\sim 3 \mathrm{~Hz}$ and $\sim 100 \mathrm{pF}$ stray capacitance). However, $R_{x x}$ is well behaved up to the turnaround point (e.g. 27 Tesla at $135 \mathrm{mK}$ ) for all temperatures measured. We limit our analysis to this regime.

There seems to be an optimum temperature for the development of any given fraction and a lowest and highest temperature cut-off for its observation. The high- $T$ limit, as usual, is a measure for its energy gap. At temperatures comparable with this gap the number of thermally excited quasi-particles becomes appreciable and flood the characteristic transport features of the FQHE. The existence of a low- $T$ limit is unusual, is not observed for higher filling factors, and characteristic of the Wigner crystal regime. The inset to Fig. 2 summarizes these limits as determined in a qualitative manner from many traces such as seen in Fig. 2. As expected, the high- $T$ limit is maximum at $\nu=1 / 5$ and $\nu=1 / 7$ and drops towards the center. This reflects the decreasing energy gap of the FQHE states in the CF model as the $\mathrm{CF}$ liquid at $\nu=1 / 6$ is approached.

In contrast to the high- $T$ limits, the low- $T$ limits show a monotonic increase with increasing $1 / \nu$ (higher $B$ field). The origin of this transport behavior at such low filling factors remains unclear, but is most probably intimately related to electron solid formation. The most likely interpretation of a low- $T$ limit for FHQE observation in Fig. 2 involves a two-phase picture in this lowfilling factor regime and, with increasing temperature, a transition from a Wigner crystal to an electron liquid, which assumes FQHE correlation at the relevant filling factors. The Wigner crystal is the $T=0$ ground state, 
whereas the different fractions of the FQHE form the ground state at elevated temperatures. This progression seems to be at odds with a simple thermodynamic argument [24]: The ground state is determined by the free energy, $F=E-T S$, where $E$ is the energy at $T=0$ and $S$ is the entropy. If the Wigner solid is the ground state at $T=0$ it is expected to remain the ground state for non-zero $T$, since its excitations are gapless and hence $T S$ grows rapidly compared to the case of a FQHE state, which is gapped. While this argument always holds for infinitesimal temperatures Price et al. [24] have shown that it fails as $T$ becomes a substantial fraction of the FQHE gap energy. At this stage the very high density of states for excitations at the edge of the FQHE gap gives rise to an exponential growth in $T S$ and wins out over $T S$ of the Wigner solid, which rises only as a power law. Hence the magnitude of the energy gap is the decisive parameter for this phase transition rather than any small difference in the ground state energies between solid and liquid.

The calculated transition temperature for the $\nu=1 / 7$ state is $\sim 600 \mathrm{mK}$ for a clean sample with parameters close to those of our specimen [24]. This value drops to $\sim 400 \mathrm{mK}$ when disorder is taken into account phenomenologically and approximate FQHE gap energies are inferred from experiment. Both values considerably exceed the low- $T$ limit of $\sim 135 \mathrm{mK}$ for $\nu=1 / 7$. However, given our very limited ability to treat disorder and its impact on transport in these highly correlated phases and given our present inability to determine experimentally the $\nu=1 / 7$ energy gap - not to mention the magnetoroton gaps, which set the energy scale for the entropy an agreement within a factor of three may be quite satisfactory. In particular, the trend of increasing disorder moving the transition to lower temperatures allows for a simple rationale to account for the discrepancy.

On the other hand, the general features of the inset of Fig. 2 seem not to be consistent with the essence of the melting calculations. According to the model, melting occurs when $T$ has reached a substantial fraction of the FQHE gap energy. Taking the high- $T$ limit of the inset to Fig. 2 as a measure for the energy gap of the FQHE liquid one would expect the lower limit - taken to reflect the melting temperature - to track the former. This is definitely not the case. In fact, both seem to be rather independent from each other: the "gaps" (high- $T$ limit) show the characteristic minimum around $\nu=1 / 6$, whereas the "melting temperature" (low- $T$ limit) rises monotonically with inverse $\nu$. Calculations for higherdenominator states than $1 / 7$ (and 1/9) may well reveal such a dependence, but, it appears, that a more general consideration may be more fruitful in uncovering the origin of the general trend seen in the inset to Fig. 2.

A simple classical Wigner crystal melting picture [26], in which the electrons are viewed as point charges on a triangular lattice yields a melting temperature of $T_{m} \sim$
$260 \mathrm{mK}$ for our sample. This temperature is in reasonable proximity to the experimental overall "melting temperature" of $\sim 120 \mathrm{mK}$ of the inset of Fig. 2. Of course, this crude calculation yields a filling factor-independent value (relatively well reflected in experiment for $1 / \nu \geq 5.5$ ) since it is independent of $B$-field, which determines the "size" of the electrons via the magnetic length. Eventually, around $\nu \geq 1 / 5$ this simple model fails totally and quantum correlations dominate, leading to the ascendance of the FQHE states.

Independent of the model used to estimate the transition temperature, melting of the electron crystal and subsequent formation of series of FQHE liquids at higher temperatures is able to describe the qualitative features of $R_{x x}$ in our experiments. For a quantitative comparison between experiment and theory much remains to be achieved and, most likely, yet higher quality 2DES specimens are required. The exceedingly high resistances of Fig. 2 are probably an indicator for the influence of remnant disorder and density fluctuations. Once the Wigner crystal has melted, one would expect for the developing FQHE states resistances in the range of $\mathrm{k} \Omega$ 's, rather than the M $\Omega$ 's, observed in Fig. 2. These high resistance values are probably due to density fluctuations leading to coexisting patches of electron crystal and electron liquid at any given $B$-field. The percolating electron path through this patchwork would make electron transport considerably more difficult. Alternatively, these high resistances may be of an intrinsic origin, in which the transition from the solid to the liquid is more complex and states at rational fractional fillings evolve gradually, carrying with them aspects of the solid that makes them more susceptible to localization.

In summary, in an exceptionally high quality sample, $R_{x x}$ minima arise at Landau level filling factors $\nu=2 / 11$, $3 / 17,3 / 19,2 / 13,1 / 7,2 / 15,2 / 17$, and $1 / 9$ as clear signatures of developing FQHE states. Each FQHE state appears only above a filling factor-specific temperature and in a certain temperature range. This behavior is well accounted for by melting of the Wigner crystal phase, expected to exist at such low filling factors, into the series of FQHE liquids prescribed by the composite fermion model.

We would like to acknowledge the staff of the NHMFL, especially E. Palm, T. Murphy, J. Pucci, and R. Smith for experimental assistance. We thank L.W. Engel, H. A. Fertig, J.K. Jain, and T. Knuuttila for helpful discussion. A portion of this work was performed at the NHMFL, which is supported by NSF Cooperative Agreement No. DMR-9527035 and by the State of Florida. D.C.T. and W.P. are supported by the AFOSR, the DOE, and the NSF. H.L.S. is supported by DOE and the W.M. Keck Foundation. 
[1] Y.E. Lozovik and V.I. Yudson, JEPT Lett. 22, 11 (1975).

[2] H. Fukuyama and P.A. Lee, Phys. Rev. B 18, 6245 (1979).

[3] For a review of recent theoretical and experimental results on Wigner crystal, see, for example, the chapters by H.A. Fertig and M. Shayegan in Perspectives in Quantum Hall Effect, S. Das Sarma and A. Pinczuk (Eds.), Wiley, New York (1996), and references therein.

[4] D.C. Tsui, H.L. Stormer, and A.C. Gossard, Phys. Rev. Lett. 48, 1559 (1982).

[5] R.B. Laughlin, Phys. Rev. Lett. 50, 1395 (1983).

[6] The Quantum Hall Effect, R.E. Prange and S.M. Girvin (Eds.), Springer, New York (1990).

[7] The Quantum Hall Effects, T. Chakraborty and P. Pietilainen, Springer, New York (1995).

[8] J.K. Jain, Phys. Rev. Lett. 63, 199 (1989).

[9] B.I. Halperin, P.A. Lee, and N.Read, Phys. Rev. B 47, 7312 (1993).

[10] R.L. Willett, Adv. Phys. 46, 447 (1997).

[11] Composite Fermions: A Unified View of the Quantum Hall Regime, O. Heinonen (Edt), World Scientific, Singapore (1998).

[12] L.W. Engel, C.C. Li, D. Shahar, D.C. Tsui, and M. Shayegan, Solid State Commun. 104, 167 (1997).

[13] P.K. Lam and S.M. Girvin, Phys. Rev. B 30, 473 (1984).

[14] D. Levesque, J.J. Weis, and A.H. MacDonald, Phys. Rev. B 30, 1056 (1984).

[15] K. Esfarjani and S.T. Chui, Phys. Rev. B 42, 10758 (1990).

[16] X. Zhu and S.G. Louie, Phys. Rev. B 52, 5863 (1995).

[17] K. Yang, F.D.M. Haldane, and E.H. Rezayi, Phys. Rev. B 64, 081301 (2001).

[18] H.W. Jiang, R.L. Willett, H.L. Stormer, D.C. Tsui, L.N. Pfeiffer, and K.W. West, Phys. Rev. Lett. 65, 633 (1990).

[19] R.R. Du, H.L. Stormer, D.C. Tsui, L.N. Pfeiffer, K.W. Baldwin, and K.W. West, Solid State Commun. 70, 2944 (1996).

[20] V.J. Goldman, M. Shayegan, and D.C. Tsui, Phys. Rev. Lett. 61, 881 (1988).

[21] J.R. Mallett, R.G. Clark, R.J. Nicholas, R.L. Willett, J.J. Harris, and C.T. Foxon, Phys. Rev. B 38, 2200 (1988).

[22] H. Buhmann, W. Joss, K.v. Klitzing, I.V. Kukushkin, A.S. Plaut, G. Martinez, K. Ploog, and V.B. Timofeev, Phys. Rev. Lett. 65, 1056 (1990); ibid. 66, 926 (1991).

[23] M. Hayne, M.K. Ellis, A.S. Plaut, A. Usher, and K. Ploog, Surface Science 263, 39 (1992).

[24] R. Price, X. Zhu, P.M. Platzman, and S.G. Louie, Phys. Rev. B 48, 11473 (1993); P.M. Platzman and R. Price, Phys. Rev. Lett. 70, 3487 (1993).

[25] R.L. Willett, H.L. Stormer, D.C. Tsui, L.N. Pfeiffer, K.W. West, and K.W. Baldwin, Phys. Rev. B 38, 7881 (1988).

[26] C.C. Grimes and G. Adams, Phys. Rev. Lett. 42, 795 (1979).

FIG. 1. Diagonal resistance $R_{x x}$ of a sample of $\mathrm{n}=$ $1.0 \times 10^{11} \mathrm{~cm}^{-2}$ and $\mu=10 \times 10^{6} \mathrm{~cm}^{2} /$ Vsec. Arrows mark several key fractional Landau level filling factors.
FIG. 2. $R_{x x}$ above $20 \mathrm{~T}$ at various temperatures. The vertical, dashed lines show the positions of the Landau level filling factors $\nu=1 / 5,2 / 11,3 / 17,3 / 19,2 / 13$, and $1 / 7$. The inset summarizes high- $T$ limits (open squares) and low- $T$ limits (solid dots) for the observation of features in $R_{x x}$ at various $\nu$. The dashed line is only a guide to the eye. The low-T limits may be viewed as the melting line from Wigner crystal to FQHE liquids and the high-T limits are measures the energy gap of FQHE states (see text for details).

FIG. 3. $R_{x x}$ of the same sample as in Fig. 2 placed in a hybrid magnet with fields up to 42 Tesla at two different temperatures. 


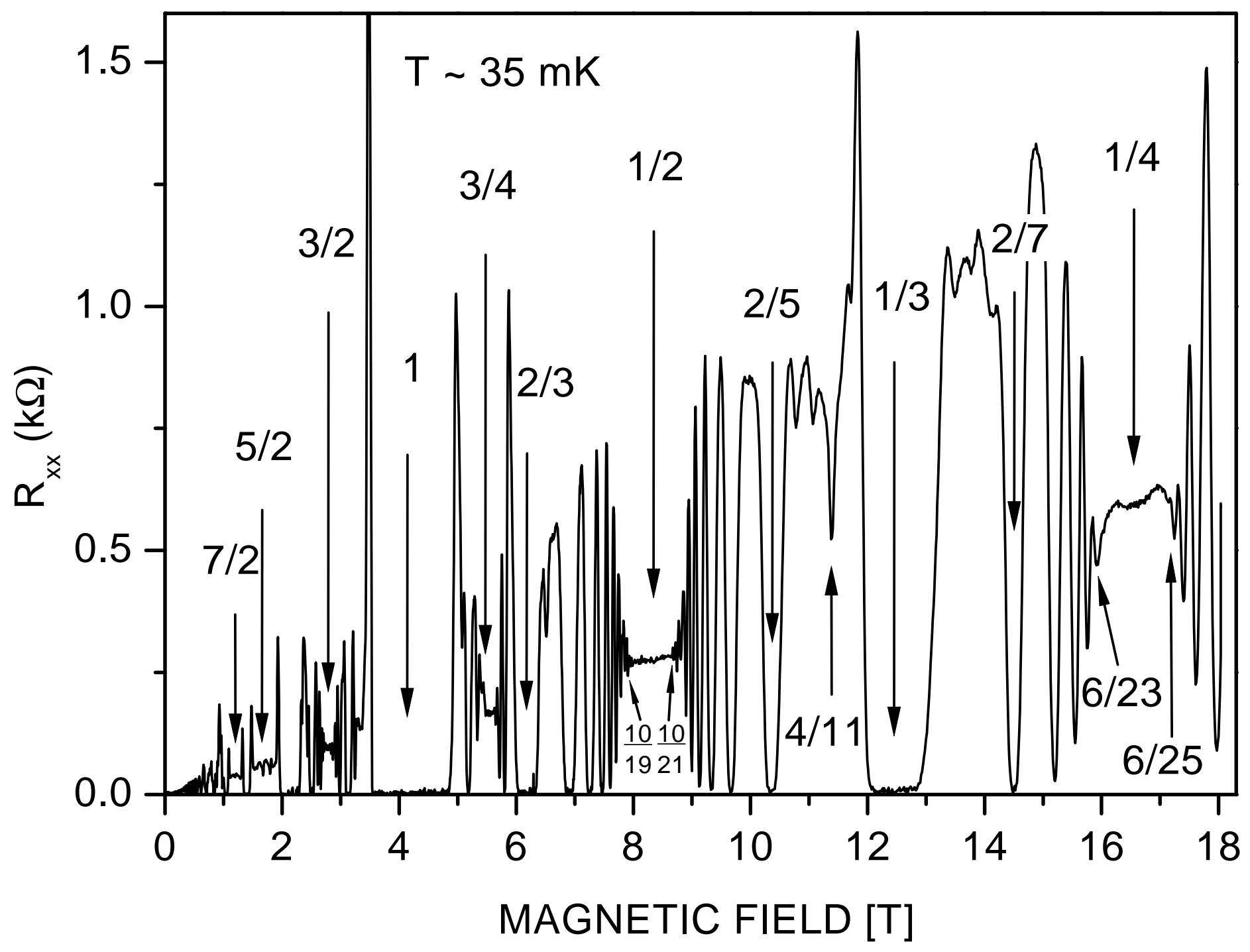




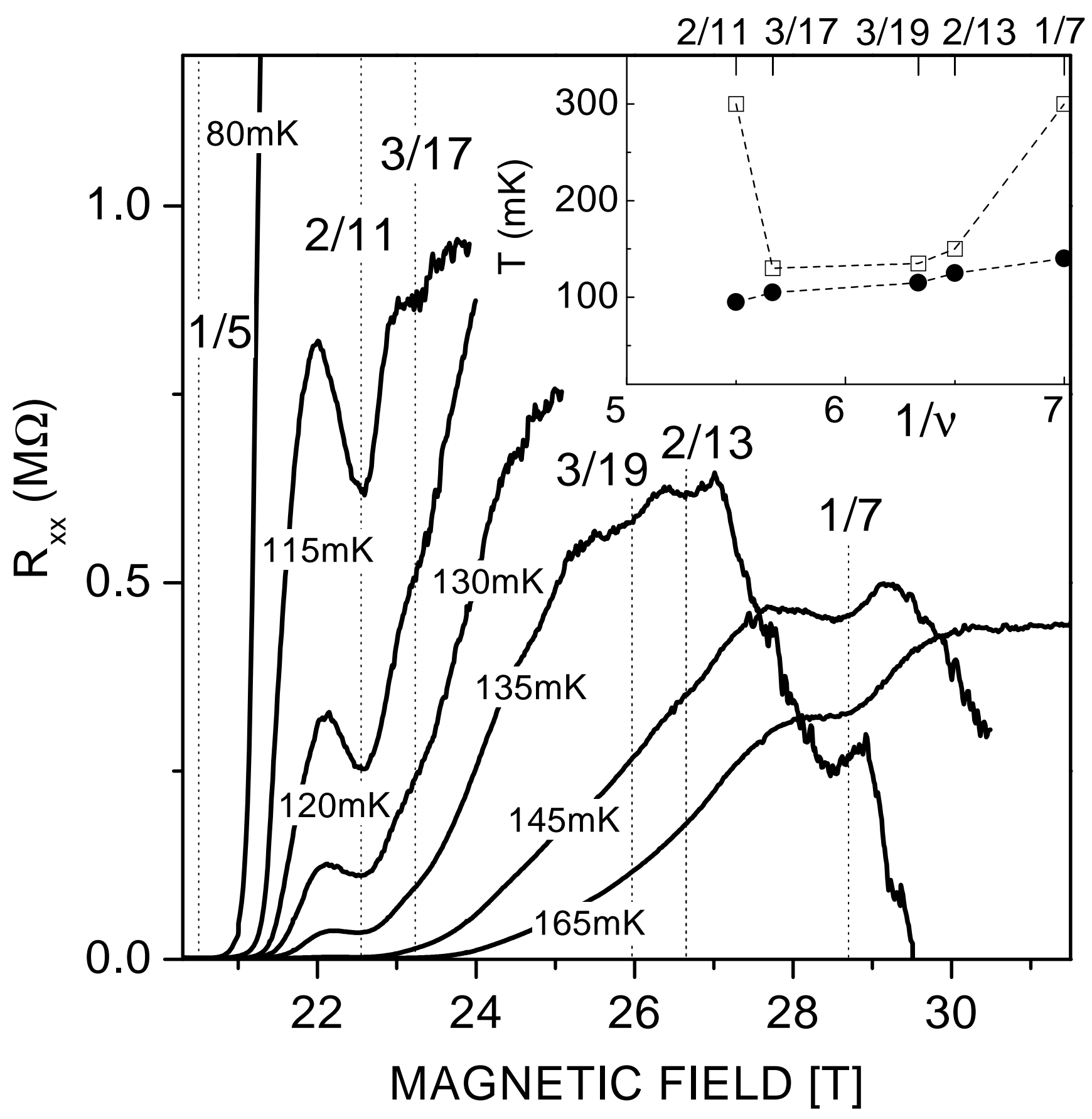




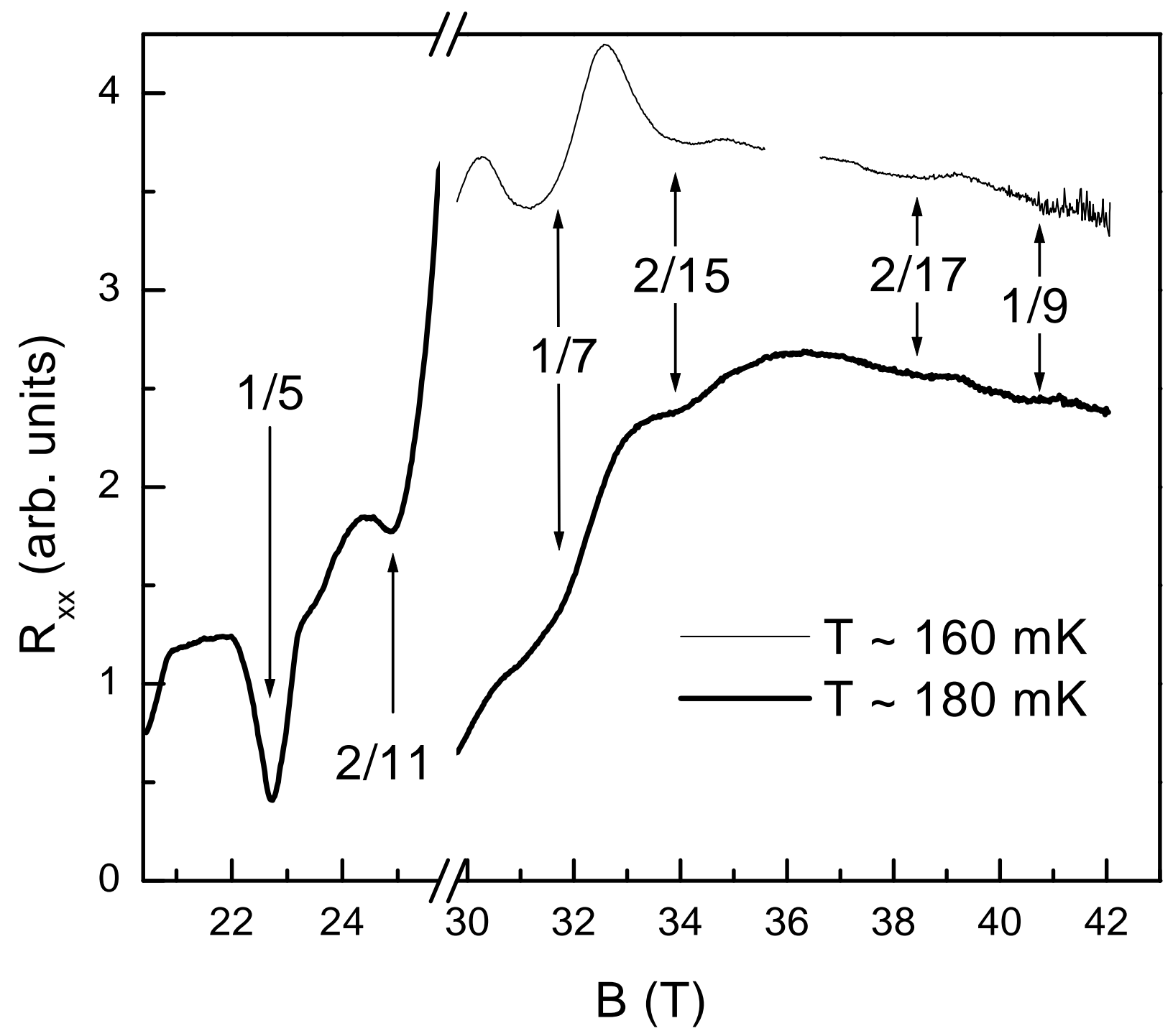

